\title{
Sympathetic Overdrive in Hypertension, The Role of Beta Blocker, Focus on Bisoprolol
}

\author{
Lucia Kris Dinarti \\ Department of Cardiology and Vascular Medicine, Faculty of Medicine, Public Health and Nursing, UniversitasGadjahMada - \\ Dr. Sardjito General Hospital, Yogyakarta, Indonesia \\ Corresponding author: \\ Lucia Kris Dinarti, MD, - email: kris_dinarti@ugm.ac.id \\ Department of Cardiology and Vascular Medicine, Faculty of Medicine, Public Health and Nursing, Universitas Gadjah \\ Mada - Dr. Sardjito General Hospital \\ Jalan Farmako Sekip Utara, Yogyakarta, Indonesia 55281
}

\section{ABSTRACT}

Sympathetic neural factors played an important role in homeostatic blood pressure control. Dysregulation in sympathetic function may favor the development and progression of the hypertensive state. Beta-blocker, as one of antihypertensive drugs, provides several positive effects to hinder overactivity of sympathetic nerve in patients with hypertension.

Keywords: sympathetic nervous system; hypertension; cardiovascular risk; target organ damage; antihypertensive drug treatment

\section{Introduction}

Autonomic nervous system has a pivotal role in modulating cardiovascular functions and in controlling blood pressure values, both at rest and in response to environmental stimuli. Hypertension, as one of most challenging condition, is considered induced by derangement of sympathetic and parasympathetic cardiovascular regulation. Appearance and sustained high blood pressure were maintained by enhanced sympathetic nerve activity and an attenuated vagal cardiac tone in animal models of hypertension. ${ }^{1}$

\section{Activity of sympathetic nervous system}

Sympathetic nervous system (SNS) is responsible to control and to maintain various body conditions such as regulatory mechanism of blood pressure, electrolyte balance, and maintenance of homeostatic state. This system is also integral part in the regulation of daily energy expenditure through the control of resting metabolic rate and thermogenesis in response to various physiological stimuli, changing states of energy, intake of food, consumption of carbohydrate, and hyperinsulinemia. Therefore, SNS overactivation is steadily related with at least two component of metabolic syndrome, i.e hypertension and obesity. ${ }^{2}$

\section{Hyperactivity of SNS in many diseases}

In obese humans with the metabolic syndrome, muscle sympathetic nerve traffic values are augmented even without hypertension. ${ }^{3}$ Muscle sympathetic nerve activity is concordant with waist circumference in individuals with the metabolic syndrome. ${ }^{3}$ In addition, during diet-induced weight loss, the decreases in sympathetic activity are accompanied by reduction in all components of the metabolic syndrome. ${ }^{4}$ In other conditions, sympathetic activity is elevated in patients with mild to moderate or end-stage renal insufficiency. ${ }^{5,6}$ This condition was also found in obstructive sleep apnea and 
chronic obstructive pulmonary diseases which lead to more predominance of sympathetic over parasympathetic activity in heart rate modulation. ${ }^{7}$

\section{Hyperactivity of SNS in hypertension}

One hypothesis stated "neurogenic nature of high blood pressure". It tried to explain that hypertensive state was affected by dysregulation in sympathetic modulation of cardiovascular function, mainly contributing in its early development and late progression. ${ }^{8}$

Sympathetic role in cardiovascular system had repeatedly studied to clarify its part in the progression of essential hypertension. One study reported that juvenile hypertension was correlated with resting tachycardia, which has hyperkinetic state. This condition might be triggered by adrenergic overdrive, observed by increased main adrenergic neurotransmitter, i.e norepinephrine in young patients. ${ }^{9}$ Other study also showed that young-stage 1 hypertensive patients which has sympathetic predominance had sustained hypertension than those who were without autonomic nervous system dysfunction after six years of follow-up. ${ }^{10}$ In addition, sympathetic activation reflected by higher resting heart rate, produce turbulent blood flow in vessels bifucartio, generate atherosclerotic plaque, and increase the stiffness of large blood vessels. ${ }^{11,12,13,14}$ Not only affecting in enhancing resting heart rate values, cardiac sympathetic drive is also associated with structural and functional modification of myocardial, such as left ventricular hypertrophy or left ventricular diastolic dysfunction, resulting in elevated morbidity and mortality if its left untreated. ${ }^{15,16,17}$

Several mechanism that are proposed to explain mechanism of hypertension-related SNS overactivity including baroreflex dysfunction, cardiopulmonar reflex dysfunction, central factors, chemoreceptor activation, involvement of insulin/leptin, and reninangiotensin system and other humoralststems. ${ }^{1}$ In regard to baroreflex dysfunction, it is hypothesized that less response of baroreceptors to blood pressure changes due to cellular impairment or a stiffening of the arterial wall may trigger sympathetic overdrive. Eventhough, in one study, it showed that in hypertensive humans, the arterial baroreflex ability to control heart rate was weaken, it still continues to effectively modulate blood pressure and sympathetic activity. ${ }^{18}$ In addition, chemoreceptor stimulation by ischemic hypoxia was considered as a sympatostimulating factor in individuals with sleep apnea, a condition frequently associated with obesity and highly prevalent in hypertension. ${ }^{19}$

Furthermore, studies from animal and human showed that insulin and leptin enhance postganglionic sympathetic drive $^{20}$ and that central and peripheral sympathostimulating effects are also induced by angiotensin $1 \mathrm{I} .{ }^{21}$ Most importantly, sympathetic nerve activity may be, either directly or indirectly, a predictor of $\mathrm{CV}$ morbidity and mortality. First, sympathetic activity is associated with and is probably a determinant of blood variability, ${ }^{22}$ which itself is a CV risk factor independent of average blood pressure values. ${ }^{23}$ Second, sympathetic hyperactivity, as measured by plasma norepinephrine, systemic norepinephrine spillover, or microneurography, is known to be an independent prognostic factor for CV-related morbid or fatal events in patients with heart failure, end-stage renal failure, major cardiac arrhythmias, obstructive pulmonary disease, or after an acute stroke. ${ }^{15,24,25,26}$

Role of beta-blockers in hypertension 
Since 1976, beta-blockers had been introduced for hypertension treatment. The various beta-blocking agents differ in terms of the presence or absence of intrinsic sympathomimetic activity (ISA), membrane stabilizing activity (MSA), $\boldsymbol{\beta} 1$-selectivity, $\boldsymbol{\alpha}$-adrenergic blocking activity, and relative potencies and duration of action. ${ }^{27}$ These drugs had proved effect to lower blood pressure and became recommended treatment option by the Joint National Committee on Prevention, Detection, Evaluation, and Treatment of High Blood Pressure. ${ }^{28}$ In addition, beta-blocking agents are considered to be an alternative first-line treatment for hypertension and are also indicated for patients having concomitant angina pectoris, arrhythmias, hypertropic cardiomyopathy, cardiomyopathy, congestive hyperdynamic circulations, essential tremor, or migraine headache. ${ }^{27}$

Despite its various beneficial effect, recent evidence showed that starting therapy of hypertension with betablockers resulted in modest reduction of cardiovascular diseases(strokes and heart attacks)and minimal or zero effects on mortality. These beta-blockers effects are inferior to those of other antihypertensive drugs. As noted that beta-blockers that was frequently studied in most randomized controlled trials were atenolol. ${ }^{29}$ Thus, to extrapolate these results to other agents in the beta-blocker class is unwarranted, because insufficient quantitave evidence (i.e outcomes data). ${ }^{30}$

One class of beta-blockers that is still frequently used is bisoprolol. This agent has $\beta 1$-blockade potency ratio tenfold higher than propranolol and moderate $\beta 1$-selectivity. Bisoprolol also has zero intrinsic sympathomimetic activity, meaning that this agent will not slightly activate beta-receptor in addition to preventing the access of natural or synthetic catecholamines to the receptor,

similar as seen in metoprolol or propanolol. $^{27}$ As mentioned above, elevated heart rate as a sign of sympathetic overdrive is a common feature in patients with prehypertension and hypertension. One proposed mechanism to explain the antihypertensive actions of beta-blockers is its ability to have effects on prejunctional beta-receptors, resulting in reduction of norepinephrine release..$^{27}$ In a study demonstrated by Julius et al, it showed that in the prehypertensive group, the heart rate remained higher than in the normotensive controls after the beta blockade using intravenous propranolol, but addition of parasympathetic blockade with atropine abolished its differences. This indicates that patients with prehypertension have an increased sympathetic and a decreased vagal tone. ${ }^{9}$

Unlike thiazide diuretic or calcium channel blockers in which sympathetic activity does not entirely return to the treatment condition, beta-blocker may reduce sympathetic activity compared with the level measured in untreated patients and improve vagal cardiac control. ${ }^{31}$ In general, beta-blockers effects on adrenergic activity in essential hypertension may include lowering heart rate, reduce plasma norepinephrine and muscle sympathetic nerve traffic, and decrease plasma norepinephrine spillover. $^{31}$

\section{Summary}

Hypertension is a condition
characterized by hyperactivity of
sympathetic nerve system. Cardiac
sympathetic drive may trigger modification
in functional and structural of myocardial,
as well as increase stiffening of large
blood vessels and generate
atherosclerotic plaque, leading to greater
morbidity and mortality. Beta-blockers is
considered to reduce sympathetic nerve
activity in patients with hypertension.




\section{References}

1. Mancia G., Grassi G. 2014. The autonomic nervous system and hypertension. Circulation Res, 114:1804-1814.

2. Verma N. 2017. Sympathetic nervous system and hypertension. Hypertens J, 3:27-36.

3. Grassi G., Dell'Oro R., QuartiTrevano F., Scopelliti F., Seravalle G., Paleari F., et al. 2005. Neuroadrenergic and reflex abnormalities in patients with metabolic syndrome. Diabetologia, 48:1359-1365.

4. Straznicky N.E., Lambert E.A., Lambert G.W., Masuo K., Esler M.D., Nestel P.J. 2005. Effects of dietary weight loss on sympathetic activity and cardiac risk factors associated with the metabolic syndrome. J Clin Endocrinol Metab, 90:5998-6005.

5. Ligtenberg G., Blankestijn P.J., Oey P.L., Klein I.H., Dijkhorst-Oei L.T., Boomsma F., et al. 1999. Reduction of sympathetic hyperactivity by enalapril in patients with chronic renal failure. N Engl J Med, 340:1321-1328.

6. Converse R.L.Jr., Jacobsen T.N., Toto R.D., Jost C.M., Cosentino F., Fouad-Tarazi F., et al. 1992. Sympathetic overactivity in patients with chronic renal failure. N Engl J Med, 327: 1912-1918

7. Taranto-Montemurro L., Messineo L., Perger E., Salameh M., Pini L., Corda L. 2016. Cardiac sympathetic hyperactivity in patients with chronic obstructive pulmonary disease and obstructive sleep apnea. COPD, 13:706-711

8. Kuchel O., Genest J. 1997. A neurogenic origin of mild highrenin essential hypertension? N Engl J Med 297:2222
9. Julius S., Krause L., Schork N.J., Mejia A.D., Jones K.A., van de Ven C., et al. 1991. Hyperkinetic borderline hypertension in Tecumseh, Michigan. J Hypertens 9:77-84.

10. Palatini P., Longo D., Zaetta V., Perkovic D., Garbelotto R., Pessina A.C. 2006. Evolution of blood pressure and cholesterol in stage 1 hypertension: role of autonomic nervous system activity. J Hypertens, 24:1375-1381.

11. Carlson N., Dixen U., Marott J.L., Jensen M.T., Jensen G.B. 2014. Predictive value of casual ECGbased resting heart rate compared with resting heart rate obtained from Holter recording. Scand J Clin Lab Invest, 74:163-169.

12. Mancia G., Bousquet P., Elghozi J.L., Esler M., Grassi G., Julius S., et al. 2007. The sympathetic nervous system and the metabolic syndrome. J Hypertens, 25:909920

13. Fox K., Borer J.S., Camm A.J., Danchin N., Ferrari R., Lopez Sendon J.L., et al. 2007. Resting heart rate in cardiovascular disease. J Am Coll Cardiol, 50:823-830.

14. Cook S., Togni M., Schaub M.C., Wenaweser P., Hess O.M. 2006. High heart rate: a cardiovascular risk factor? Eur Heart J, 27:23872393.

15. Cohn J.N., Levine T.B., Olivari M.T., Garberg V., Lura D., Francis G.S., et al. 2984. Plasma norepinephrine as a guide to prognosis in patients with chronic congestive heart failure. $\mathrm{N}$ Engl $\mathrm{J}$ Med, 311:819-823.

16. Brunner-La Rocca H.P., Esler M.D., Jennings G.L., Kaye D.M. 2001.Effect of cardiac sympathetic nervous activity on mode of death 
in congestive heart failure. Eur Heart J, ;22:1136-1143.

17. Grassi G., Mark A., Esler M. 2015. The sympathetic nervous system alterations in human hypertension. Circ Res, 13;116:976-990.

18. Grassi G., Cattaneo B.M., Seravalle G., Lanfranchi A., Mancia G. 1998. Baroreflex control of sympathetic nerve activity in essential and secondary hypertension. Hypertension, 31:68-72..

19. Somers V.K., Mark A.L., Abboud FM. 1988. Sympathetic activation by hypoxia and hypercapniaimplications for sleep apnea. Clin Exp Hypertens, :413-422.

20. Mark A.L., Correia M., Morgan D.A., Shaffer R.A., Haynes W.G. 1999. State-of- the-art-lecture: obesity-induced hypertension: new concepts from the emerging biology of obesity. Hypertension, 33:537-541

21. Saino A., Pomidossi G., Perondi R., Valentini R., Rimini A., Di Francesco L., et al. 1997. Intracoronary angiotensin II potentiates coronary sympathetic vasoconstriction in humans. Circulation, 96:148-153.

22. Grassi G. 2009. Assessment of sympathetic cardiovascular drive in human hypertension: achievements and perspectives. Hypertension, 54:690-697.

23. Mancia G. 2012. Short-term and long-term blood pressure variability. In: Berbari AE, Mancia $G$, eds. Special Issues in Hypertension. Milan: Springer, pp.91-102.

24. Sander D., Winbeck K., Klingelhöfer J., Etgen T., Conrad
B. 2001. Prognostic relevance of pathological sympathetic activation after acute thromboembolic stroke. Neurology, 57:833-838.

25. Zoccali C., Mallamaci F., Parlongo S., Cutrupi S., Benedetto F.A., Tripepi G. 2002. Plasma norepinephrine predicts survival and incident cardiovascular events in patients with end-stage renal disease. Circulation, 105:13541359

26. Andreas S., Haarmann H., Klarner S., Hasenfuss G., Raupach T. 2014. Increased sympathetic nerve activity in COPD is associated with morbidity and mortality. Lung, 192:235-241.

27. Frishmann W.H. 2007. $\beta$-Blockers in hypertension. Hypertension: $A$ Companion to Braunwald's. Elsevier.

28. Chobanian A.V. Bakris G.L., Black H.R., Cushman W.C., Green L.A., Izzo J.L.Jr., et al. 2003. Seventh report of the Joint National Committee on Prevention, Detection, Evaluation, and Treatment of High Blood Pressure. Hypertension, 42:1206-1252.

29. Wiysonge C.S., Bradley H.A., Volmink J., Mayosi B.M., Opie L.H. 2017. Beta-blockers for hypertension. Cochrane Database Syst Rev, 1:1-93.

30. Ram C.V. 2010. Beta-blockers in hypertension. Am J Cardiol, 106: 1819-1825.

31. Grassi G. 2004. Counteracting the sympathetic nervous system in essential hypertension. Curr Opin Nephrol Hypertens, 13:513-519. 